\title{
A Study on the Application of Electronic Nose Coupled with DFA and Statistical Analysis for Evaluating the Relationship between Sample Volumes versus Sensor Intensity of Agarwood Essential Oils Blending Ratio
}

\author{
Sahrim Lias ${ }^{1, *}$, Nor Azah Mohamad Ali $^{2}$, Mailina Jamil ${ }^{3}$, Mohd Shafik Yuzman Tolmanan ${ }^{4}$ and Muhamad Afizzul \\ Misman $^{5}$ \\ ${ }^{1-4}$ Natural Products Division, Forest Research Institute Malaysia (FRIM), 52109 Kepong Selangor, Malaysia \\ ${ }^{5}$ Forestry and Environmental Division, Forest Research Institute Malaysia (FRIM), 52109 Kepong Selangor, Malaysia
}

\begin{abstract}
The exquisite agarwood oils are primary used for perfumery industries either as pure essential oils or in a perfume base. Commonly, Agarwood oils are extracted from low grade $100 \%$ agarwood chips via distillation processes and the extracted oil is called as pure agarwood essential oil which containing $100 \%$ of extracted material. In perfumery industry, the agarwood pure oils are often blend with other essential oils such as geranium, sandalwood, gurjum balsam, jasmine and Ylang ylang to create rich, complex and pleasant oils compared to pure Agarwood oils smell alone that may not suit all users preferences. To dates, agarwood oil quality assessment is typically carried out manually via human olfactory system which produces different results and inconsistency from traders and buyers. From the results, multiple linear regression analysis used to run the multiple regression prediction models using combination of 11 sensors shown better results by increasing the $\mathrm{R}^{2}$ value from 0.674 to 0.915 and the RMSE value from $14.65 \%$ to $6.80 \%$ compared to single regression prediction models using sensor LY2/G. The sensors intensity values from multiple sensors are showing a strong correlation to the volume of the $\mathrm{B} 1$ in the blended samples (M11 M20) as the ratio of B1 is increased.
\end{abstract}

\section{Introduction}

Agarwood is originated from Aquilaria (Thymelaeceae) species, also known as Gaharu, Jin-Koh, Chim-Hyuang, oud and Kanankohis a highly valuable fragrant wood used for incense, perfume, traditional medicines and religious ceremony. In Peninsular Malaysia, five species of Aquilaria have been recorded and they are $A$. malaccensis, A. microcarpa, A. hirta, A. beccariana and A. rostrate [1]. Among this five species, A. malaccensis and $A$. crassna is the best known species which produced high quality and multipurpose used of gaharu resin.

Usually, Agarwood is distilled for essential oils using hydrodistillation, water distillation and supercritical fluid carbon dioxide extraction (SFE) techniques. The presence of complex chemical compounds can be identified using the headspace solid phase micro extraction (HS-SPME), Gas Chromatography Mass Spectrometry (GC/GCMS) and Gas Chromatography-olfactometry (GC-O) while Electronic Nose (EN) which mimic human nose is used to detect the strong and unique heavy scent of Agarwood oil [2]. Among them, combination of EN and HS-SPMEGCMS is also used to classify the quality of four agarwood oil into high and low grades [3]. Current study revealed that the presence of sesquiterpenes and its chromonederivaties such as 10-epi- $\gamma$-eudesmol, $\beta$ agarofuran, $\alpha$-agarofuran and $\gamma$-eudesmol can be used as a standard in order to identify the high or low quality of agarwood oils [4]. High quality of agarwood oil contained major constituted of guaiene and eudesmanesesquiterpenes [5]. Two less known compounds: 9-11-eremophiladien-8-one and oxoagarospirol always found in low quality of agarwood oil. $\beta$-agarofuran, $\alpha$-agarofuran and 10 -epi- $\gamma$-eudesmol were found less in low quality Agarwood oil. Some discovered compounds like $\beta$-agarofuran, 11-dien-9-one, camphor and (-)-selina-3 are major compounds contributing to the fresh and sweet aroma which represent the gorgeous and elegant character of agarwood oil [6].

The price range and quality of Agarwood oils are very reliant on its physical properties, color, odor and consumer perceptions. High quality oil commonly is dark color, long lasting odor, expensive and categorised as either supreme or deluxe oils with the price range between USD126 to USD633 per tola while the wood price for low qualities are USD19 per kg. Agarwood wood chips are traded according to its grade. Different countries graded or classified the Agarwood oil by their own methods. In Japan, Agarwood oil is categorized as high and low quality using its chemical profiles.

* Corresponding author: sahrim@frim.gov.my 
KananKoh is categorized as the highest quality and Jinkoh is the lowest quality [7]. However, different grading techniques make the price of Agarwood oil fluctuates and not standardized in the market. Beside the grading techniques, agarwood oil itself is difficult to differentiate either it is pure or mixture oil.

Currently, there are many devices or instruments used to capture odor or scent and one of the equipment used is Electronic Nose (EN). EN is a device designed to mimic human nose functions which is to detect and differentiate smell with higher sample repeatability compared to human nose. EN being used widely for different usage such as for measurement for mango homogenate and whole fruit, mango fruit maturity, saccharomyces cerevisiae strains for alcoholic fermentation, milk self-life determination, virgin olive oil quality, zingiberaceae family oils [8-13]. DFA has been used to classify growing cell populations between the four groups defined in the learning cell population [14]. It is also used to classify Agarwood oils into pure and mixture clusters for easy observation [15] and compared to k-NN kfold classifier classification system [16].

This paper proposed a technique to observe significant data trend and to evaluate the relationship patterns between sample volume and sensor intensity from a set of Agarwood essential oil mixture samples. The blending process is based on the specific increment ratio of agarwood oil volume whereby the Gurjum balsam oil volume is fixed. All experiment and data analysis were conducted via EN experiments coupled with DFA and statistical analysis using R software.

\section{Materials and Methods}

This work is completed by conducting experiment using commercial FOX4000 EN from Alpha-MOS (Toulouse, France) bundled with AlphaSOFT version 12 data analysis software. Each of EN experiments are started with the samples preparation processes, EN parameters setup, sample heating using heating block, headspace generated manually injected to the EN using gas tight syringe and recording the sensors response data into the main database. Overall flows of this paper are as in Figure 1. The process is started with samples selection process whereby both sample were subjected to the chemical test using Gas chromatography-mass spectrometry (GCMS) equipment and chemical constituent detection technique. This test can identify each sample is Agarwood and Gurjum balsam essential oils because of each pure essential oils having only major significant chemical compounds known consisted in each oils. The process is continue with oils samples blending process, sample preparation process for EN experiments, features extraction process, data visualization and pattern recognition and classifier using DFA. Detail steps of each process are explained in the next subsection.

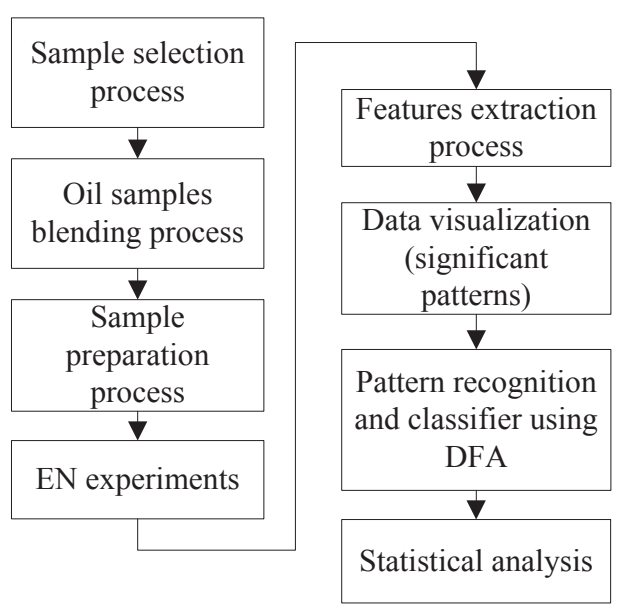

Figure. 1. Overall research flows.

\subsection{Sample Preparation}

Agarwood and Gurjum balsam essential oils samples were obtained from the Natural products division, FRIM essential oil collection whereby they were initially bought from trusted agarwood traders. 2 essential oils name as base B1 (pure agarwood) and base B2 (pure Gurjum balsam) were selected as reference essential oils. 10 mixture oils were blended from both base oils which are M11, M12, M13, M14, M15, M16, M17, M18, M19 and M20. The samples are names corresponding to the increment of B1 volume in the blended sample. Details samples preparation process, blending ratio, and pictures of blended oil samples are as in Table 1, Table 2 and Figure2.

Table 1. Sample preparation parameters.

\begin{tabular}{ll}
\hline \multicolumn{2}{c}{ Samples preparation } \\
\hline Vial size and type & $\begin{array}{l}10 \mathrm{ml} \text { glass vial with magnetic } \\
\text { clamp cap }\end{array}$ \\
Vial per sample & 5 \\
Sample volume & $\pm 10 \mu 1$ per vial (no dilution) \\
Sample code & B1, B2, M11 M20 \\
Sample blending ratio & 10 samples: B1 varies $1 \sim 10:$ \\
& B2 (fixed) \\
\hline
\end{tabular}

Table. 2. Pure Agarwood B1 and pure Gurjum B2 balsam sample blending ratio.

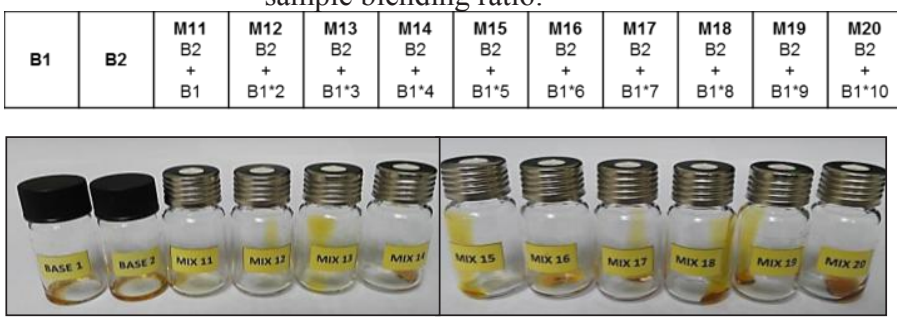

Figure 2. A set of 2 pure B1: pure Agarwood and B2: pure Gurjum balsam with 10 of blended oils (M11, M12, M13, M14, M15, M16, M17, M18, M19 and M20). 


\subsection{Electronic Nose Experiment}

EN equipment comprised of 18 metal oxide semiconductors (MOSs) chemical sensors. The EN sensor chambers are divided into 3 type of high performance (HiP) chambers; Chamber A: doped metal oxide sensors (T30/1, P10/1, P10/2, P40/1, T70/2 and PA2), chamber B: doped metal oxide sensors (P30/1, $\mathrm{P} 30 / 2, \mathrm{P} 40 / 2, \mathrm{~T} 40 / 2, \mathrm{~T} 40 / 1$ and TA2) and chamber CL2:undoped metal oxide sensors (LY2/AA, LY2/G, $\mathrm{LY} / \mathrm{gCT}, \mathrm{LY} 2 / \mathrm{gCT} 1, \mathrm{LY} 2 / \mathrm{Gh}$ and LY2/LG) which can detect flammable gases, organic compounds, toxic gases and oxidizing gases. The carrier gas used is purified air grade $(P=5 \mathrm{psi})$. The complete EN system (manual injection configuration) consisted of heating block, main EN chamber and computer installed with AlphaSOFT software.

The internal EN hardware operations flows are illustrated as in Figure 3. The injection port is the main sample inlet to the EN hardware. Each headspace generated from the heated sample is injected into the injection port with special gas tide syringe. The system will allow the purified air as the headspace carrier gas to carry the sample headspace through all 3 chambers in the system and exit at the exhaust port. Simultaneously, the AlphaSOFT software will record all EN raw data for database establishment.

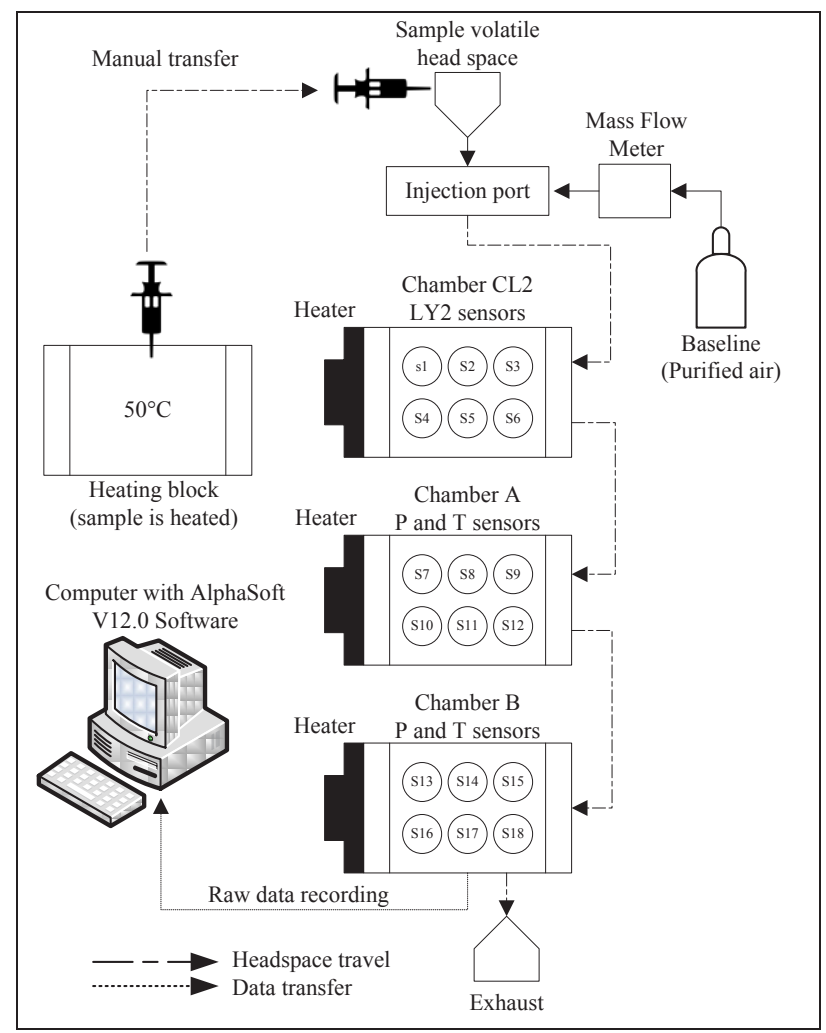

Figure 3. Alpha-MOS FOX4000 EN internal structures and sample injection flows.

EN was use to capture the uniqueness of agarwood essential oils samples scent and transform the information into sensor responses data. A total of 60 sample vials were used in the EN experiment for the database development process. A specific EN experiment protocol is optimized prior conducting the experiment. The optimized parameters used in the EN experiment are tabulated in Table 3.

Table 3. Electronic nose analytical conditions.

\begin{tabular}{|c|c|}
\hline \multicolumn{2}{|c|}{ EN experiment parameters } \\
\hline Injection type & $\begin{array}{l}\text { Manual injection } \\
\text { configuration }\end{array}$ \\
\hline Sequence type & Interlace each sample \\
\hline Acquisition duration (sec) & 120 \\
\hline Acquisition period (sec) & 1 \\
\hline Data sampling (sec) & 1 \\
\hline Acquisition time (sec) & 600 \\
\hline Flow (ml/min) & 150 \\
\hline Gas tight syringe volume (ml) & 2.5 \\
\hline Injection volume $(\mu \mathrm{l})$ & 500 \\
\hline Injection speed $(\mu \mathrm{l} / \mathrm{sec})$ & 500 \\
\hline Heating temperature $\left({ }^{\circ} \mathrm{C}\right)$ & 50 \\
\hline Heating time ( $\mathrm{sec}$ ) & 300 \\
\hline Library construction option & $\begin{array}{l}\text { Maximum (automatic } \\
\text { selection of signal extrema). } \\
\text { Delta }(\mathrm{Ro}-\mathrm{R}) / \mathrm{Ro} \text { ( } \mathrm{R} \text { : } \\
\text { resistance difference divided } \\
\text { by the Ro: initial resistance). } \\
\text { In this mode, the displayed } \\
\text { value is relative resistance }= \\
\text { (Ro- } \mathrm{R}) / \mathrm{Ro} \text {, where } 0 R \text { is the } \\
\text { resistance at } \mathrm{t}=0 \text { (baseline } \\
\text { resistance) and } \mathrm{R} \text { is the } \\
\text { resistance at time } \mathrm{t}\end{array}$ \\
\hline
\end{tabular}

\subsection{Data analysis (features extraction, DFA model and $R$ statistical analysis)}

All raw data were observed and extracted using AlphaSOFT version 12 data analysis software provided with the instrument. All experiment raw data were gathered from all 18 sensors responses. Each vial producing 120 continuous data corresponding to 120 seconds of acquisition duration with $1 \mathrm{~s}$ sampling rate. The "Delta R/R0" option displays the sensor values as relative resistance variation whereby $\mathrm{R}$ is sensor response correspond to the sample injected and RO is the initial resistance value as a baseline (no sample injected). For the main EN database, the "Maximum (automatic selection of signal extrema)" option criteria is selected. Therefore, with this selection, only the maximum peak data (+ve peak and -ve peak) from each sensor with a total of 18 data per vial were extracted. These databases comprised of $60 \times 18$ data size were then used for the development of DFA model.

Statistical analyses were performed using $\mathrm{R}$ software to test the relationship between sensor's sensitivity and volumes of Agarwood oils blending ratio. Mann-Whitney $U$ test was tested on the maximum peak data to compare the differences between B1 and M11 M20 with 95\% confident level for each sensor. The P-value is used as the main indicator and if the value is smaller than 0.05 , it is indicates that the sensor can differentiate between B1 and M11 M20. Simple linear regression analysis was then applied to the maximum peak data of each sensor to find which sensor gave better 
result in predicting the volumes of Agarwood. Higher rsquare $\left(R^{2}\right)$ value indicates better result. For better prediction, multiple linear regression approach was also applied to the maximum peak data for the development of model, which combines several selected sensors to predict the volume of Agarwood in the blended oils. Finally, Analysis of Variance (ANOVA) was used to compare between the best models for single sensor with the model with a combination of several sensors.

\section{Results}

The raw data are extracted using maximum peak point from each sensor. All 18 sensor data are selected using library construction option: maximum for data library construction as one of example data in Figure 4 pointed as "Maximum response" which produced a total of $60 \times 18$ data matrix.

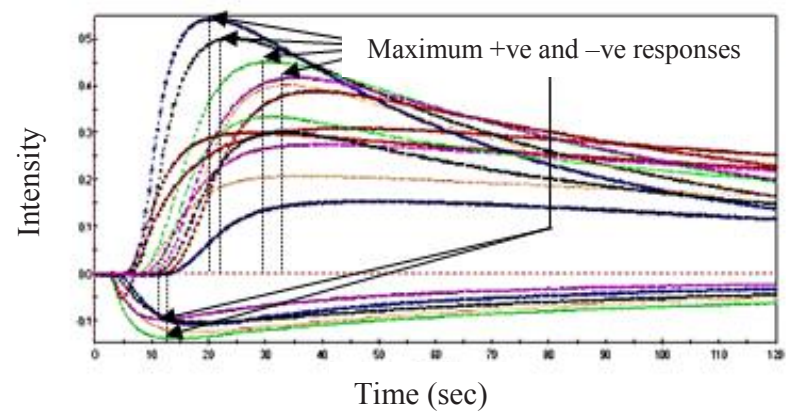

Figure 4. Total 120 seconds data recording time per sample with maximum response $+v e$ and - ve points for each sensor extracted as raw data

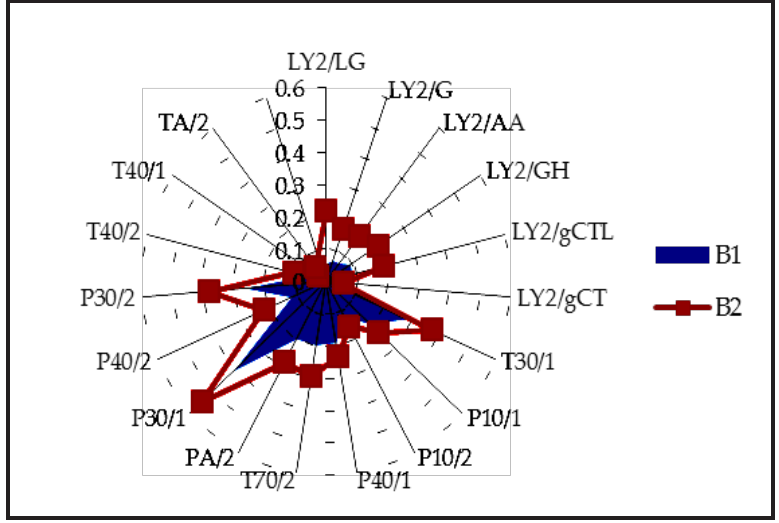

Figure 5. Radar view of $18 \mathrm{EN}$ sensor data comparison between B1 and B2 samples.

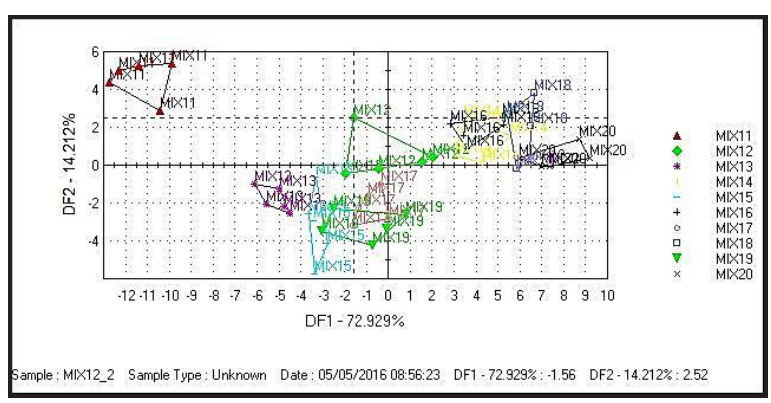

(a)

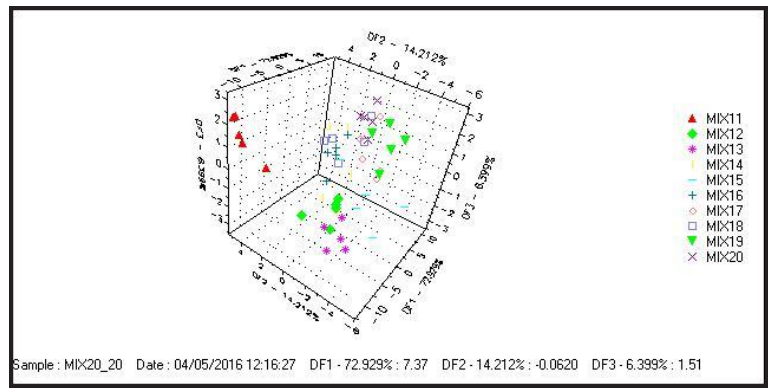

(b)

Figure 6. DFA model for 10 samples M11, M12, M13, M14, M15, M16, M17, M18, M19 and M20: (a) DFA1 and DF2 view and (b) DFA1, DF2 and DF3 view.

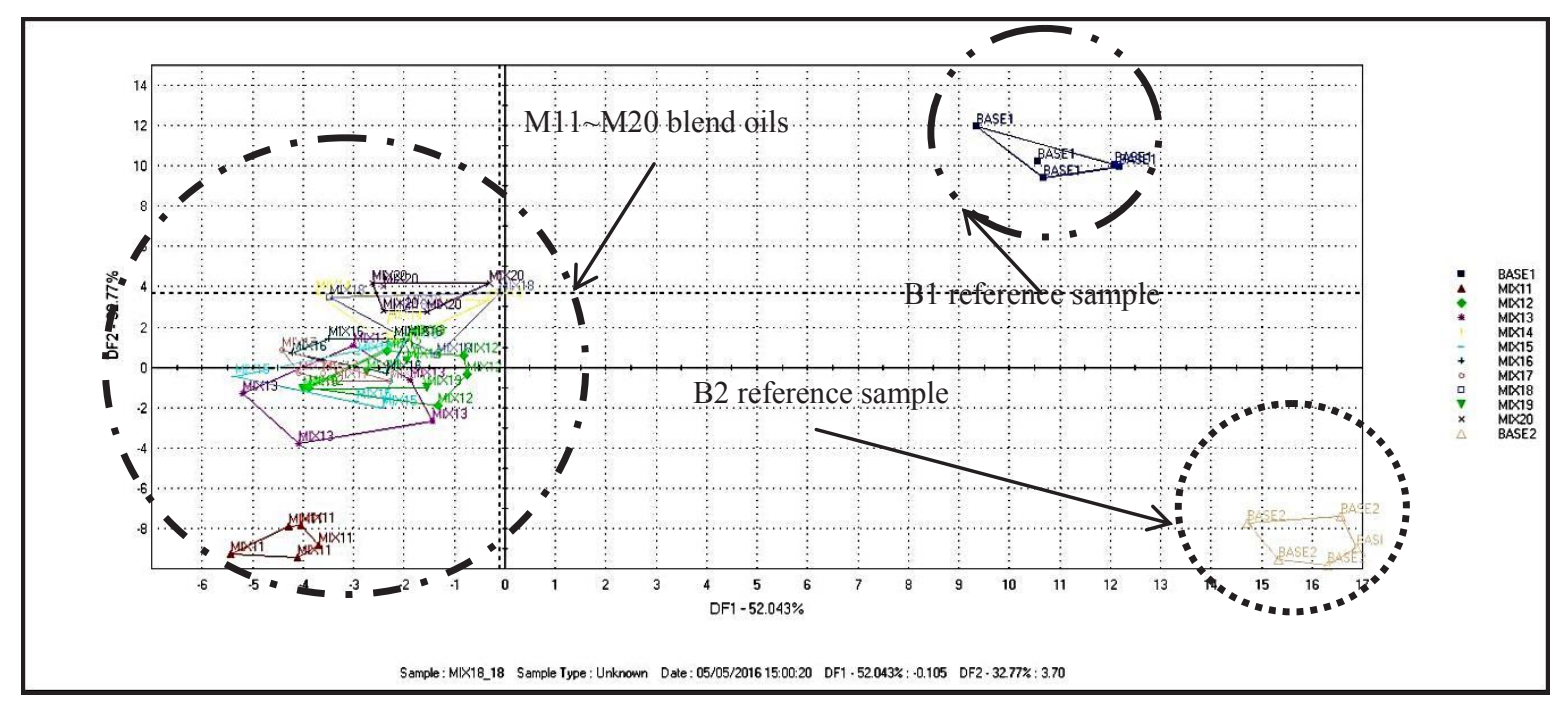

Figure 7. DFA model for 12 samples B1, B2, M11, M12, M13, M14, M15, M16, M17, M18, M19 and M20. 


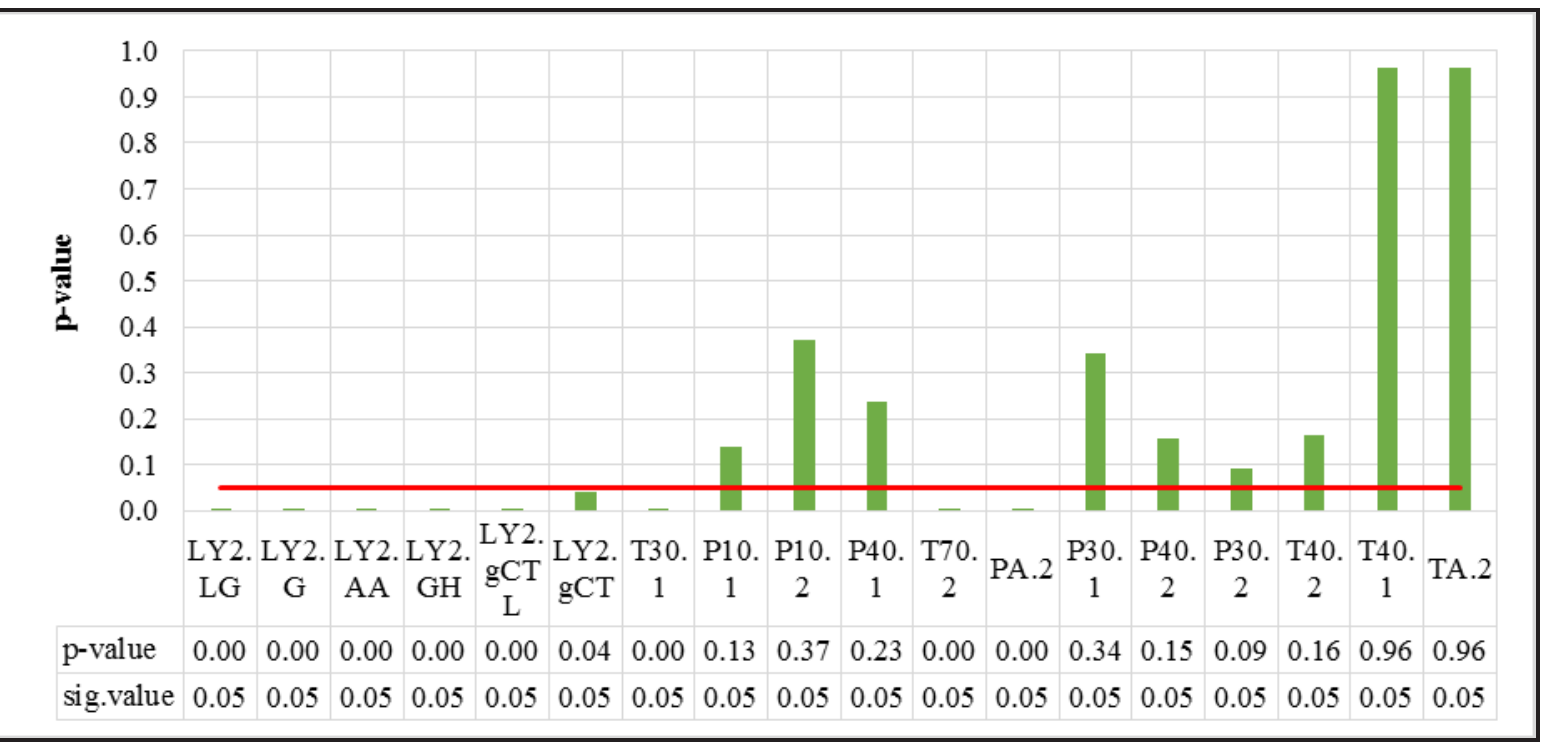

Figure 8. The p-value of Mann-Whitney U test tested on each sensor. Value over 0.05 indicates non-significant different between pure and mix Agarwood oils.

\section{Discussion}

Figure 5 shows that sample B1 and sample B2 are having a different EN sensors data response and patterns intensity whereby sample B2 is having higher sensors response value compared to sample $\mathrm{B} 1$. This proven that $\mathrm{B} 1$ and $\mathrm{B} 2$ are different samples and having a different fragrance. $\mathrm{B} 1$ and $\mathrm{B} 2$ then is blended with specific ratio into 10 blended samples (M11 M20). All 12 samples are then subjected into the DFA model constructed including B1 and B2 labeled as reference sample. There are 3 main group clusters (cluster 1: B1, cluster 2: B2 and cluster 3: M11 M20) can be observed in Figure 7 showing that each of group is having a different sensor response.

The observation is further done within the blended samples (M11 M20) for any sensors intensity differences. After they are subjected into constructed DFA model by excluding B1 and B2, they can be observed as different sample in 3D view $(\mathrm{DFA} 1=72.929 \%, \quad \mathrm{DF} 2=14.212 \%$ and $\mathrm{DF} 3=6.399 \%$ view) much better compared to $2 \mathrm{D}$ view (DFA $1=72.929 \%$ and DF $2=14.212 \%$ ) as in Figure 6 (b) and (a) respectively.

A statistical test, particularly Mann-Whitney U test has shown that 9 sensors including LY2/LG, LY2/G, LY2/AA, LY2/GH, LY2/gCTL, LY2/Gct, T30/1, T70/2 and $\mathrm{PA} / 2$ did pass the test at $95 \%$ confident level as shown in Figure 8. B1 and 10 blended samples (M11 M20) Agarwood oils at minimum concentration of 50 percent can be differentiate using these sensors. Other sensors, namely as $\mathrm{P} 10 / 1, \mathrm{P} 10 / 2, \mathrm{P} 40 / 1, \mathrm{P} 30 / 1$, $\mathrm{P} 40 / 2, \mathrm{P} 30 / 2, \mathrm{~T} 40 / 2, \mathrm{P} 30 / 2, \mathrm{~T} 40 / 2, \mathrm{~T} 40 / 1$ and TA/2 have shown non-significant results. As for model development for the prediction of Agarwood volumes in mix oils, in overall most sensors gave low correlation values with an average $\mathrm{R}^{2}$ of 0.20 and Root Mean Square Error (RMSE) of $22.45 \%$. Only five sensors, which are LY2/LG, LY2/G, LY2/AA, LY2/GH, and LY2/gCTL gave moderate correlation values. LY $2 / \mathrm{G}$ gave the best predictions of Agarwood volumes in mix oils with $\mathrm{R}^{2}$ and RMSE of 0.674 and $14.65 \%$ respectively. The prediction of Agarwood volumes in mix oils using model developed by multiple regression analysis approach has shown a significant improvement with the combination of LY2/G, T30/1, PA/2, LY2/AA, LY2/LG, P30/1, P30/2, LY2/gCTL, LY2/GH, T70/2 and P40/2's sensors. The $\mathrm{R}^{2}$ value of combined sensors has improved to 0.915 . This model has also successfully reduced the RMSE to $6.80 \%$. The result of ANOVA test was also shown the significant different between the best model using single sensor and combined sensors.

\section{Conclusions}

As a conclusion, from the EN experiments conducted, DFA model is enabled to differentiate B1 and B2 into two different clusters. Multiple linear regression analysis used to run the multiple regression prediction models using combination of 11 sensors had shown better results by increasing the $\mathrm{R}^{2}$ value from 0.674 to 0.915 and the RMSE value from $14.65 \%$ to $6.80 \%$ compared to single regression prediction models using sensor LY2/G. From the results, it can be concluded that the sensors intensity values from multiple sensors are having a strong correlation to the volume of the $\mathrm{B} 1$ in the blended samples (M11 M20) as the ratio of B1 is increased.

Acknowledgments: All support from Forest Research Institute Malaysia (FRIM), Asia Pacific Association of Forestry Research Institutions (APAFRI) and Natural Products Division staffs are very much appreciated. 


\section{References}

1. Chang, Y.S., NorAzah, M.A. \& Abu, S.A. Gaharu: A prized incense from Malaysia. Malaysian Oil Science \& Technology 2001, vol. 9(2), pp. 26-27.

2. Sahrim, L., Nor Azah, M.A., Mailina, J., Muhd Hafizi, Z., Abdul Majid, J. \& Nurlaila, I. Classification of Agarwood Oils Using K-NN KFold. Sensor Letters 2014, vol. 12(6-7), pp. 10381040.

3. Nurlaila, I., Nor Azah, M.A., Mailina, J., Mohd Hezri, F.R., SaifulNizam, T. \&Mohd Nasir, T. A. review study of Agarwood oil and its quality analysis. Jurnal Teknologi 2014, vol. 68(1), pp. 3742.

4. Nor Azah, M.A., Nurlaila,I., Mailina,J., Mohd Nasir,T. \&MohdHezri, F.R. Analysis of aroma compounds from Gaharu oil by Head-space solid phase microextraction and Gas chromatographymass spectrometry. In proceeding of the Seminar on Medicinal \& Aromatic Plants: Translating natural products $R, D \& C$ initiatives in line with economic transformation programme 2012, Pp: 180-185.

5. Ishihara, M., Tsuneya. \&Uneyama,K. Components of the Agarwood smoke on heating. Journal of Essential Oil Research 1993, vol. 5, pp. 419-423.

6. Pripdeevech, P., Khummueng,W. \&Park,S.K. Identification of odoractivecomponents of Agarwood essential oils from Thailand by Solid Phase Microextraction-GC/MS and GC-O. Journal of Essential Oil Research 2011, vol. 23, Pp.46-53.

7. Ishihara, M., Tsuneya. \&Uneyama,K, Components of the volatile concentrate of Agarwood. Journal of Essential Oil Research 1993, vol. 5, pp. 283-289.

8. A. R. Paper Development of Electronic Nose Measurement for Mango (Mangifera Indica) Homogenate and whole fruit. Proc. Fla. State Hort. Soc 2004, vol. 51, no. 7, pp. 421-425.

9. M. Lebrun, A. Plotto, K. Goodner, M. Ducamp, and E. Baldwin. Discrimination of mango fruit maturity by volatiles using the electronic nose and gas chromatography. PostharvestBiology and Technology 2008, vol. 48, pp. 122-131.

10. P. Chalier, D. Chevalier-lucia, C. Ghommidh, and J. A. Ragazzo-sanchez, "Identification of Saccharomyces cerevisiae strains for alcoholic fermentation by discriminant factorial analysis on electronic nose signals", Electron. J. Biotechnol 2010, vol. 13, no. 4 .

11. S. Bazzo, S. Cade, and E. Chanie, "Shelf life determination by electronic nose: application to milk," Sensor and Actuators 2005, vol. 106, pp. 199-206.

12. D. L. García-gonzález and R. Aparicio, "Coupling MOS sensors and gas chromatography to interpret the sensor responses to complex food aroma: Application to virgin olive oil," Food Chem. 2010, vol. 120 , no. 2, pp. 572-579.

13. Sahrim, L., Nor Azah, M.A., Mailina, J., Azrina, A., Siti Humeirah A.G. and Mohammad Faridz Z. P. Classification of selected essential oil from family zingiberaceae using e-nose and discriminant factorial analysis (dfa) techniques: an initial study. Journal of Applied Mechanics and Materials (AMM) 2015, pp. 799-800: 932-936.

14. J. Savatier, J. Vigo, and J. M. Salmon, "Monitoring cell cycle distributions in living cells by videomicrofluorometry and discriminant factorial analysis," Cytometry Part A 2003, vol. 56A, pp. 814.

15. Sahrim, L., Nor Azah, M.A., Mailina, J., Muhd Hafizi, Z. and Siti Humeirah A.G., "Classification of pure and mixture Agarwood oils by Electronic Nose and Discriminant Factorial Analysis (DFA)," 2015 International Conference on Smart Sensors and Application (ICSSA) 2015, pp.7-10.

16. Sahrim, L., Nor Azah, M.A., Mailina, J., Abdul Majid, J. and Mohd Fadhli, O. Discrimination of pure and mixture agarwood oils via electronic nose coupled with k-NN kfold classifier. Procedia Chemistry 2016, vol. 20, p 63-68. 